\title{
ARTICLE \\ Clozapine use in old age psychiatry
}

\author{
Amey Kirrane, Biswadeep Majumdar \& Anna Richman
}

Amey Kirrane is a core trainee on the Health Education North West training programme Biswadeep Majumdar and Anna Richman are both consultants in old age psychiatry at Mossley Hill Hospital, Liverpool. Correspondence Dr Amy Kirrane, V7 Building, Kings Business Park, Prescot, Liverpool, L34 1PJ, UK. Email: ameykirrane@doctors.net.uk

\section{Copyright and usage}

(C) The Royal College of Psychiatrists 2018

\section{SUMMARY}

Clozapine is one of the most effective drugs available to psychiatrists for treating psychosis. It is currently licensed for use in treatment-resistant schizophrenia and psychosis in Parkinson's disease, but its use in old age psychiatry is very uncommon. With the ageing population, and the increased incidence of psychosis in older patients, it is important to consider whether this is a drug that is not being used to its full advantage.

\section{LEARNING OBJECTIVES}

- Appreciate the differences in titration and monitoring of clozapine in older adults, compared with working-age adults

- Consider the efficacy of clozapine in older people and its impact on mortality

- Understand the side-effect profile of clozapine in older adults

\section{DECLARATION OF INTEREST}

None.

A survey undertaken by Paranthaman $\&$ Baldwin (2006) showed that specialists in old age psychiatry were not familiar with or confident in using clozapine in older patients, particularly because of the lack of available evidence on its safety. In the same survey, 61 consultants (45\% of responders) had patients who were on clozapine and 39 had started at least one patient on the medication.

\section{Use in treatment-resistant schizophrenia}

It is estimated that $1 \%$ of the population will experience schizophrenia in their lifetime (Royal College of Psychiatrists 2015). The National Institute for Health and Care Excellence (NICE) guidelines recommend the use of clozapine in patients whose schizophrenia remains unresponsive to treatment despite trialing at least two different antipsychotic medications, one of which should be an atypical antipsychotic (NICE 2014). Similar to their younger counterparts, older patients with a new diagnosis of schizophrenia will experience hallucinations, delusions and, to a lesser extent, thought disorder (Tune 2003). Those who are diagnosed with schizophrenia in early adult life, but develop a chronic course as they grow older, tend to have more problems with negative symptoms. Where older patients differ from younger adults is in their response to pharmacological treatment, which can be unpredictable and variable. Some antipsychotics show serious side-effects such as cerebrovascular events (Kohen 2010).

\section{Use in Parkinson's disease}

Around $0.2 \%$ of the UK population will be affected by Parkinson's disease in their lifetime, with the average age at onset at 60 years (http://www.parkinsonsdiseaseearlysymptoms.com/tag/parkinsonsdisease-statistics). Psychosis is seen in the advanced stages and it therefore follows that antipsychotics will more likely be needed in older patients. These patients are very likely to have comorbidities and to be subject to polypharmacy, which it is particularly important to consider. NICE guidelines on the management of psychotic symptoms in Parkinson's disease advise reducing the use and dose of antiparkinsonian medications and avoiding typical antipsychotics (NICE 2017). However, if the benefits of an antipsychotic outweigh the risks, then the guidelines state that clozapine should be the medication of choice for treating psychosis. There are two common causes of psychotic symptoms in Parkinson's disease. The first is an iatrogenic side-effect of antiparkinsonian drugs and the second is organic processes in the brain. Between 20 and $30 \%$ of people with Parkinson's disease will develop psychotic symptoms, with an increased prevalence seen in the later stages of the disease (Kuzuhara 2001).

\section{Titration}

In older adults (>65 years of age), the mean therapeutic dose for clozapine is $25-100 \mathrm{mg} /$ day (Gareri 2008): by comparison, the mean therapeutic dose for working-age adults is 300-600 mg/day. Higher doses may be found in patients who were started on clozapine in adulthood and progressed to old age services while still on it. It is usual for higher doses to be split into twice daily administration, with the larger dose in the evening. This relates to the side-effects profile of the drug, which includes sedation and orthostatic hypotension, and it helps reduce the risk of falls in older patients. There is no specially devised titration regime to be used in older adults, only the general advice of increasing doses slowly and using the minimum effective dose (Table 1). If a patient responds 
adequately to a low dose then there is no rationale to trial higher doses. General advice is that rapid titration can lead to unwanted side-effects.

\section{Monitoring}

Current guidelines on commencing and monitoring clozapine are detailed in Box 1. Blood tests are used to monitor for agranulocytosis, the incidence and fatality rate of which has greatly reduced since the introduction of blood monitoring (from 0.3 to 0.01\% (eMC 2017)). Patients undergoing titration are also required to have physical health observations at set intervals, pre- and post-dose, using the Lester Positive Cardiometabolic Health Resource (the Lester Tool). Blood tests are used to monitor for metabolic syndrome and electrocardiograms (ECGs) to check for prolonged QTc interval or signs of any other developing abnormalities. In terms of monitoring clozapine and norclozapine plasma levels, neither NICE nor the Maudsley Prescribing Guidelines (Taylor 2015) give any guidance on frequency, but some individual trusts suggest annual monitoring. There are no separate guidelines for monitoring in older patients.

BOX 1 Guidelines on commencing and monitoring clozapine in older adults $(>65$ years of age)

Clozapine should be started at a dose of $12.5 \mathrm{mg}$ once daily

Blood pressure, temperature and pulse should be monitored hourly for first $6 \mathrm{~h}$

ECGs should be done at baseline and after dose increases. Once stabilised, patients should be offered an ECG annually Neutrophil count should be monitored as follows:

- weekly for the first 18 weeks of treatment

- at 2-week intervals between weeks 18 and 52

- after 1 year of treatment, if neutrophil counts are stable, patients may be monitored at 4-week intervals

- monitoring must continue throughout treatment and for at least 4 weeks after discontinuation

If the patient is tolerating clozapine, the dose can be increased by $25-50 \mathrm{mg}$ each day, until a dose of $300 \mathrm{mg} /$ day is reached

Further dose increases should be made slowly in increments of 50-100 mg each week

A plasma level of $350 \mu \mathrm{g} / \mathrm{L}$ should be aimed for to ensure an adequate trial, but response may occur at a lower plasma level

(Taylor et al, 2015)
TABLE 1

Clozapine prescribing for treatment-resistant schizophrenia and Parkinson's disease in older adults ( $>65$ years of age)

\begin{tabular}{|cll|}
\hline & \multicolumn{1}{|c|}{ Treatment-resistant schizophrenia } & Parkinson's disease \\
\hline Starting therapy & $\begin{array}{c}6.25-12.5 \mathrm{mg} / \text { day } \\
\text { Dose should be increased by no more } \\
\text { than } 6.25-12.5 \mathrm{mg} \text { once or twice } \\
\text { a week }\end{array}$ & $6.25 \mathrm{mg} /$ day \\
\hline $\begin{array}{c}\text { Therapeutic } \\
\text { dose range }\end{array}$ & $\begin{array}{c}50-100 \mathrm{mg} / \mathrm{day} \\
\text { Maximum dose }\end{array}$ & $25-37.5 \mathrm{mg} /$ day \\
\hline Ending therapy & $\begin{array}{c}\text { Gradual reduction over 1-2 weeks } \\
\text { (in non-emergencies) }\end{array}$ & $\begin{array}{c}\text { Gradual reduction by steps of } 12.5 \mathrm{mg} \\
\text { for a period of at least } 1 \text { week } \\
\text { (preferably 2 weeks) }\end{array}$ \\
\hline & &
\end{tabular}

Source: Taylor et al (2015).

\section{Pharmacokinetics and pharmacodynamics of clozapine}

Clozapine differs from other antipsychotics in its receptor binding profile. As a rule, antipsychotics work on $\mathrm{D}_{2}$ dopamine receptors, but clozapine's affinity for $\mathrm{D}_{4}$ receptors is ten times greater (Sanyal 1997). This becomes a significant factor when considering neuroleptic malignant syndrome (NMS), which is the result of central $\mathrm{D}_{2}$ receptor blockade. In addition, it is well known among specialists that the risk of NMS is higher in those with Parkinson's disease or Lewy body dementia, because of central dopamine blockade, and therefore antipsychotic use should be avoided if possible. If an antipsychotic is necessary, clozapine is one of the best options because of its lower affinity for $\mathrm{D}_{2}$ receptors. It also has a greater specificity for the limbic system, compared with other antipsychotics, hence its low incidence of extrapyramidal adverse effects and its usefulness in Parkinson's disease. Further information is detailed in Box 2.

\section{BOX 2 Pharmacokinetics of clozapine}

- Clozapine is a tricyclic dibenzodiazepine derivative

- $27-50 \%$ of a dose reaches systemic circulation

- Levels peak at $2.1 \mathrm{~h}$ if administered twice daily

- The drug's half-life ranges from 6 to $33 \mathrm{~h}$

- Steady state concentration is reached in 7-10 days

- The volume of distribution is $1.6 \mathrm{~L} / \mathrm{kg}$

- $95 \%$ is bound to plasma proteins

- It is metabolised through hepatic enzymes (CYP1A2 and CYP3A4)

- Clozapine is broken down into norclozapine and clozapine- $\mathrm{N}$-oxide: these are pharmacologically active with a similar action to clozapine but with weaker effect and shorter duration

- $50 \%$ is excreted in the urine and $30 \%$ in the faeces (Curran \& Bullock, 2005) 
It is worth considering the changes in the drug's pharmacokinetics in older adults. Increased volume distribution and decreased hepatic clearance will likely mean that older people do not metabolise clozapine as efficiently as working-age adults, leading to higher plasma levels and putting patients at increased risk of side-effects (Crooks 1976).

\section{Contraindications}

Box 3 lists contraindications to clozapine use in older adults.

\section{Mortality}

\section{Treatment-resistant schizophrenia}

In schizophrenia, the mortality rate is four times that of the general population, with statistics suggesting that people with schizophrenia die 10 years earlier than the general population (Parks 2006). Capasso et al (2008) carried out a 55-year follow-up study looking at the causes of mortality in schizophrenia and the most common were found to be cardiovascular, followed by cancer. This is consistent across several studies. Clozapine is the most effective drug at lowering mortality in schizophrenia, as shown in an 11-year follow-up study (Tiihonen 2009).

Pridan et al (2015) looked specifically at clozapine use in patients aged 60-80+ in a retrospective analysis and found the mortality rate to be $18 \%$. This is compared with a mortality rate of $18.6 \%$ with other antipsychotics, suggesting that clozapine

BOX 3 Contraindications to clozapine use in older adults ( $>65$ years of age)

- Hypersensitivity to the active substance

- Patient unable to undergo regular blood tests

- History of toxic or idiosyncratic granulocytopenia/agranulocytosis (with the exception of chemotherapy)

- History of clozapine-induced agranulocytosis

- Clozapine treatment must not be started concurrently with substances known to have a substantial potential for causing agranulocytosis; concomitant use of depot antipsychotics is to be discouraged

- Impaired bone marrow function

- Uncontrolled epilepsy

- Alcoholic or other toxic psychoses, drug intoxications, comatose conditions

- Circulatory collapse and/or CNS depression of any cause

- Severe renal or cardiac disorders

- Active liver disease associated with nausea, anorexia or jaundice; progressive liver disease; hepatic failure

- Paralytic ileus

(DrugBank 2016) treatment is safe in older patients with treatmentresistant schizophrenia.

\section{Parkinson's disease}

In Parkinson's disease, modern medicine usually means that life expectancy is not limited, but in advanced stages quality of life can become poor because of the unpleasant symptoms. Treating patients with antipsychotics often means a fine balance between reducing psychosis and preserving motor function. Hack et al (2014) studied 36 patients (mean age 68.3 years) with Parkinson's disease on clozapine and found a mortality rate of $8.6 \%$ over an 8-year period. However, the authors indicated that the deaths were not directly related to clozapine use, but resulted from complications of the disease.

\section{Polypharmacy}

As already noted, people over 65 years of age often have multiple comorbidities and are therefore subject to polypharmacy. Research found that $45 \%$ of older adults attending an emergency department were taking five or more medications and 11\% are taking ten or more (Banerjee 2011). A balance of under- and overprescribing is needed to avoid prescribing cascades and there are now several tools available to review a patient's medications and identify any drugs that are unnecessarily contributing to polypharmacy. One that has been used widely across general practice is STOPP/START (Screening Tool of Older People's Prescriptions/ Screening Tool to Alert to Right Treatment), for which there is a strong evidence base (Gallagher 2008). It consists of a list of 65 STOPP criteria and 22 START criteria, and has good interrater reliability. This is a tool that psychiatrists might want to use before commencing clozapine in an older patient, to help reduce the risk of drug interactions. Examples are shown in Table 2. Clinicians might also want to consider the effect of prescribing other psychotropics while a patient is on clozapine. The British National Formulary (BNF) highlights that commonly used antidepressants, in particular fluoxetine and paroxetine, can lead to an increase in plasma clozapine levels, and therefore risk potential toxicity (Joint Formulary Committee 2017). Sertraline has a similar effect, but to a lesser degree. Clozapine use with lithium can increase the risk of extra-pyramidal side-effects and neurotoxicity, and serious adverse events have been reported with concomitant use of clozapine and benzodiazepines.

\section{Efficacy}

\section{Treatment-resistant schizophrenia}

Clozapine has been shown to be the most effective drug in treating schizophrenia, with research 
TABLE 2 STOPP/START examples of medication review in older adults ( $>65$ years of age)

\begin{tabular}{|lll|}
\hline Medications & Circumstances prompting review & Reason for review \\
\hline Anticholinergics & To treat extra-pyramidal side-effects of antipsychotic medication & Risk of anticholinergic toxicity, including confusion and urinary retention \\
\hline Benzodiazepines & $>1$ month use of long-acting benzodiazepines \\
If patient has fallen in the past 3 months & Risk of prolonged sedation, confusion, impaired balance and falls \\
\hline SSRls & $\begin{aligned} \text { If sodium level has been }>130 \text { in the past } 2 \text { months } \\
\text { Citalopram and escitalopram: prolonged QT interval }\end{aligned}$ & $\begin{array}{l}\text { SSRls can cause/worsen hyponatraemia } \\
\text { Do not use citalopram and escitalopram in patients with congenital QT } \\
\text { syndrome or known pre-existing QT interval prolongation } \\
\text { Do not use citalopram and escitalopram in combination with other drugs } \\
\text { known to prolong the QT interval }\end{array}$ \\
\hline
\end{tabular}

STOPP/START, Screening Tool of Older People's Prescriptions/Screening Tool to Alert to Right Treatment; SSRI, selective serotonin reuptake inhibitor.

suggesting that it helps in up to $30-50 \%$ of those who have failed to respond to other antipsychotics (Kane 1988). One study (Sajatovic 1998) looked at clozapine's clinical effectiveness in 329 patients. Interestingly, they found a bigger therapeutic advantage in those aged 55 to 64 years, compared with those aged 65 years and older, with mean improvements of 19.8\% (55 to 64) and 5.7\% (over $65)$ on the Brief Psychiatric Rating Scale (BPRS). Pridan et al (2015) found that clozapine was generally well tolerated, with a reduction in hospital readmission rates, and found no difference in dose or response between patients aged 60-69 years and those aged 70-79 years. Nobody discontinued clozapine because of side-effects and it had no impact on existing comorbidities.

\section{Parkinson's disease}

Efficacy in Parkinson's disease has been confirmed by Pollack et al (2004), who found an improvement on the Clinical Global Impressions (CGI) scales and Positive and Negative Syndrome Scale (PANSS) and reported that over half of their patients had recovered completely from their psychotic symptoms by the end of the 5 -year follow-up study. The average age of the sample in the clozapine group was 71.2 years. Adverse effects observed were reported to be mild to moderate and could be controlled with slower titration. Similar findings were reported by the Parkinson Study Group (1999). The mean dose of clozapine used was $24.7 \mathrm{mg}$ (s.d. $=6.25-50 \mathrm{mg})$. Again, they found improvements in the CGI, BPRS and the Scale for the Assessment of Positive Symptoms (SAPS). However, at the end of this double-blind trial, all 53 participants were allowed to trial clozapine and 6 subsequently died. The authors concluded that these deaths were not related to clozapine: three of the patients were nursing home residents and two had advance directives in place. A further study supporting efficacy (Klein 2003) looked at 32 individuals (mean age 73 years) with Parkinson's disease over a 5-year period. Only 3 participants stopped clozapine because of side-effects and 1 for personal reasons; 19 individuals were able to continue, with improvement in baseline rating scales. The remaining 9 stopped clozapine because of improvement in symptoms, which did not return after ceasing medication.

\section{Adverse effects}

The Clinical Antipsychotic Trials of Intervention Effectiveness (CATIE) showed that the majority of patients were unlikely to adhere to their prescribed antipsychotic medication because of intolerable side-effects (Lieberman 2005). When compared with the other atypical antipsychotics in the CATIE study, clozapine had a significantly longer time to discontinuation (median $=10.5$ months), suggesting that individuals were better able to tolerate its side-effects. Those on olanzapine, risperidone and quetiapine stayed on their medication for only 2-3.5 months. It is worth taking this into consideration in the older population, who are more vulnerable to side-effects.

Barak et al (1999) reviewed the use of clozapine in older adults: 133 patients were observed, only 15 of whom reported side-effects. In addition, there were 7 cases of agranulocytosis or leukopenia. The authors concluded that clozapine was safe and effective in older patients.

\section{Agranulocytosis}

One of the more serious risks of clozapine use, and therefore the most monitored, is agranulocytosis. A reduction in white blood cells could ultimately lead to a severe infection, sepsis or death. One study (Alvir 1993) of 11555 individuals on clozapine found that the risk ratio for agranulocytosis with increasing age was 1.06 (95\% CI 1.05-1.08). Neither the dose of clozapine nor the plasma levels seemed to predict the increased risk of agranulocytosis, and it therefore appears that age-related changes in clozapine clearance may account for the increased incidence in older people. Along with the increased 
incidence with age, this study also confirms that there is an increased incidence in women, with a risk ratio of 1.60 (95\% CI 0.99-2.58).

There have been several studies of drug-induced agranulocytosis in older people for a wide range of drugs, including clozapine. The suggested mortality for an older patient with drug-induced agranulocytosis is $20 \%$, with causes of death usually including sepsis, shock or pneumonia (Andrès 2014). Several factors indicate a poor outcome. Along with age and septic shock, these include the presence of metabolic syndromes such as renal failure. Therefore, this must be considered when starting clozapine in an older patient. Guenette et al (2013) studied 48 individuals, aged 65 and over, on clozapine and found 6 cases of mild neutropenia, but no participants met the criteria for agranulocytosis. However, the rate of neutropenia (12.5\%) was much higher than previously reported.

\section{Cardiac side-effects}

Regular clinical observations are taken while patients are titrated onto clozapine, usually pre- and postdose. As well as checking temperature, this is to monitor for changes in heart rate and blood pressure. This is significant in older people because of an agerelated decline in noradrenaline levels, which contributes to lower blood pressure (Salzman 2005). Therefore, orthostatic hypotension is expected to be more common in older patients than in their working-age counterparts and particular attention needs to be paid to polypharmacy, as the cumulative hypotensive effect of other prescribed medications is likely to contribute to dizziness and falls. The impact of orthostatic hypotension can be reduced by using lower doses of clozapine and a slow titration.

Among the most commonly used drugs in Parkinson's disease are dopamine agonists, a sideeffect of which is hypotension. The additive effect with clozapine could put patients at increased risk of dizziness, fainting and falls. Hypotension can also be a symptom of Parkinson's disease itself and is a further contributory factor.

Tachycardia is caused by the effect of clozapine on the autonomic nervous system (Lally 2014). If tachycardia is confirmed as benign, a beta blocker can be used to control the heart rate. Again, this is the recommendation for working-age adults, and there are no guidelines on the use of beta blockers in older adults. In 2016, Lally and colleagues attempted a Cochrane review of evidence for pharmacological interventions for clozapine-induced sinus tachycardia (Lally 2016). However, no randomised controlled trial matched their inclusion criteria and they were therefore unable to comment on the efficacy of betablockers.
Another adverse effect of clozapine is myocarditis, of which tachycardia is a warning sign (Lally 2014). This is thought to be due to a hypersensitivity reaction. One study (Ronaldson 2011) found that the onset of myocarditis was usually within the first 5 weeks of commencing clozapine. The same authors suggested obtaining baseline troponin and C-reactive protein (CRP) levels and monitoring these at regular intervals, advising that a significant increase in CRP and an elevation in troponin has a $100 \%$ sensitivity for detecting clozapine-induced myocarditis in patients who are experiencing symptoms (malaise, breathlessness, chest pain). Furthermore, clinicians should be aware of the development of cardiomyopathy, which tends to come weeks to months later on after clozapine initiation. There have been single case reports documenting cardiac side-effects of clozapine in older adults, but no studies on a larger scale exist. It is therefore difficult to comment on whether more serious adverse events such as myocarditis and cardiomyopathy happen more frequently in older people, but it is likely that the mortality rate would be higher.

\section{Metabolic syndrome and diabetes}

We know that prevalence rates of diabetes and hypertriglyceridemia are higher in older people owing to age-related changes. It is well documented that clozapine causes significant increases in plasma glucose and triglyceride levels compared with other antipsychotics (Lamberti 2006; Novartis Pharmaceutical Corporation 2013), so it adds to the existing risk factors in older adults. Physicians should work to optimise diabetes control and other risk factors, such as cholesterol and triglyceride levels, to help reduce the long-term risk of cardiovascular events.

\section{Anticholinergic side-effects}

Compared with other antipsychotics, clozapine is considered to be highly anticholinergic (Gareri 2008). Peripheral symptoms, to which older people are much more sensitive, are detailed in Table 3. A large study (Cancelli 2010) involving 6912 individuals aged 65 years and over on anticholinergics found that they were at an increased risk of cognitive decline and dementia. The risk improved when the suspect drug was stopped.

Polypharmacy guidelines published by Scottish Government Model of Care Polypharmacy Working Group (2015) state that prescribed medications with anticholinergic properties are contributing to increased morbidity and mortality. They also give advice on how to assess and reduce the anticholinergic burden that includes use of the Modified Anticholinergic Risk Scale (mARS). 
TABLE 3 Receptor profile of clozapine and related side-effects

\begin{tabular}{|ll|}
\hline Receptor & Side-effects \\
\hline Histamine $\mathrm{H}_{1}$ & Weight gain \\
& Sedation \\
\hline Alpha-1 adrenergic & Sedation \\
& $\begin{array}{l}\text { Orthostatic hypotension } \\
\text { Reflex tachycardia }\end{array}$ \\
\hline Alpha-2 adrenergic & Peripheral blockade can increase cardiac output \\
\hline Serotonin 5 -HT $1 \mathrm{C}$ & Attenuated hyperprolactinaemia \\
& Weight gain \\
\hline Muscarinic & Delirium \\
& Confusion \\
& Cognitive deficits \\
& Blurred vision \\
& Dry mouth \\
Urinary retention \\
Constipation \\
Might promote development of tardive dyskinesia \\
\hline
\end{tabular}

Source: Gareri et al (2008)

Another side-effect due to clozapine's anticholinergic properties is constipation. Constipation should be treated quickly and effectively because of the risk of bowel obstruction and perforation, which could prove fatal. The choice of laxative should be tailored to each patient, considering their medical history and other prescribed medications. Constipation is a common occurrence in older people and can become particularly troublesome if not acted on promptly (Medicine and Healthcare Products Regulatory Agency 2017).

There are no large-scale studies looking specifically at the effect of clozapine's anticholinergic properties in older people.

\section{Hypersalivation}

This is considered a common side-effect and it carries a high risk in older patients, given the possibility of aspiration and infection (Table 4). In general, older people are at an increased risk of aspiration pneumonia for a multitude of reasons, including polypharmacy and stroke. Various conservative methods are suggested to reduce the risk of aspiration pneumonia, but pharmacological treatment of hypersalivation in older people is limited. Atropine drops 1\%, ipratropium bromide spray and pirenzepine show limited statistical significance (Praharaj 2006). More commonly used drugs such as hyoscine hydrobromide should be avoided in older people because of their potential effect on cognition.

Individuals who have Parkinson's disease with hypersalivation need to be given special consideration, as they are at increased risk of swallowing and choking difficulties. Specialist advice from a speech and language therapist should be sought.

\section{Over-sedation and falls}

\section{Conclusions}

TABLE 4 Frequency of commonly reported side-effects of clozapine (18-65 years)

\begin{tabular}{|lc|}
\hline Side-effect & Frequency, \% \\
\hline Hypersalivation & 47.8 \\
Sedation & 45.9 \\
Weight gain & 31.3 \\
Dizziness & 26.9 \\
Constipation & 25.1 \\
Insomnia & 20.0 \\
Nausea and vomiting & 16.5 \\
Dyspepsia & 13.8 \\
Weakness & 7.5 \\
Urinary incontinence & 6.5 \\
Dry mouth & 5.1 \\
Dysarthria & 4.8 \\
Postural (orthostatic) hypotension & 4.4 \\
Akathisia & 4.4 \\
Diabetes & 3.3 \\
Syncope & 3.1 \\
Convulsions & 2.5 \\
Muscle rigidity & 0.2 \\
\hline
\end{tabular}

Source: Herbert et al (2003).

There are no large-scale studies of the risk of clozapine-related hypersalivation in older adults.

Sedation is the most common reason for clozapine discontinuation in older patients (Naranjo 1995). It is often a short-lived side-effect, but can persist in older patients, therefore contributing to increased levels of confusion and risk of falls. This risk could be reduced by administering clozapine at bedtime.

In Parkinson's disease there are a multitude of reasons a patient might fall - gait freezing (motor blocks), muscle weakness, hypotension and blurred vision are just a few. Therefore, careful assessment of the risk of falls is required. Pollack et al (2004) studied the efficacy of clozapine in Parkinson's disease and found that a dose no higher than $50 \mathrm{mg} /$ day was optimal for reducing psychotic symptoms without worsening motor symptoms. The Parkinson Study Group (1999) found mean improvements in baseline scores without worsening of parkinsonian symptoms when clozapine was used.

From the evidence base on clozapine use in people over 65 , which is not extensive, the drug appears to be as effective in that age group as it is in younger adults. Older people can generally tolerate clozapine well and it is a valuable treatment option to reduce the disease burden of psychosis and improve quality of life. Data from large-scale studies are lacking, but from the evidence that is 
MCO answers

$1 c \quad 2 d \quad 3 a \quad 4 b \quad 5 b$ available, clozapine does not appear to significantly increase mortality. As in younger adults, there is a long list of possible side-effects that may affect an older patient, and each case needs to be considered individually to identify which side-effects the person may be vulnerable to and appropriate plans put in place to monitor and reduce these. Using low doses and a slow titration remains appropriate and is the safest way to monitor for emerging sideeffects and to identify the lowest therapeutic dose.

There is not enough information and data to develop detailed guidelines and protocols for clozapine titration and monitoring in older people. It is also worth considering the practicalities, cost and impact of in-patient admission for titration onto clozapine and whether this puts patients at unnecessary risk of hospital-acquired infection etc. Some of the studies discussed in this article have included people aged 75 years and over, but usually only one or two participants in each case, and therefore extra caution should be taken in this age group.

To aid the development of age-specific guidelines for older adults, multicentre trials should be carried out to look specifically at clozapine use in those over the age of 65 . With dedicated guidelines, clinicians will feel more confident in prescribing clozapine to older people, with the hope that they can have a longer periods of disease stability and are able to remain living independently in the community for longer.

\section{References}

Alvir JM, Lieberman JA, Safferman AZ, et al (1993) Clozapine-induced agranulocytosis. Incidence and risk factors in the United States. New England Journal of Medicine, 329: 162-7.

Andrès E, Mourot R, Keller 0, et al (2014) Drug-induced agranulocytosis in elderly patients: diagnosis and management of life-threatening infections and septic shock. Journal of Infectious Diseases \& Therapy, 2: 191.

Banerjee A, Mbamalu D, Ebrahimi S, et al (2011) The prevalence of polypharmacy in elderly attenders to an emergency department: a problem with a need for an effective solution. International Journal of Emergency Medicine, 4: 22.

Barak Y, Wittenberg N, Naor S, et al (1999) Clozapine in elderly psychiatric patients: tolerability, safety and efficacy. Comprehensive Psychiatry, 40: 320-5.

Cancelli I (2010) Anticholinergic drugs increase the risk of cognitive decline and dementia in older people. Evidence-based Mental Health, 13: 44.

Capasso RM, Lineberry TW, Bostwick JM, et al (2008) Mortality in schizophrenia and schizoaffective disorder: an Olmsted County, Minnesota cohort: 1950-2005. Schizophrenia Research, 98: 287-9.

Crooks J, O'Malley K, Stevenson IH (1976) Pharmacokinetics in the elderly. Clinical Pharmacokinetics, 1: 280-96.

Curran S, Bullock R (2005) Practical Old Age Psychopharmacology. Radcliffe Publishing.

DrugBank (2016) Clozapine. DrugBank (http://www.drugbank.ca/drugs/ DB00363).

Electronic Medicines Compendium (2017) Clozaril 25mg and 100mg Tablets. eMC (https://www.medicines.org.uk/emc/medicine/32564).

Gallagher P, Ryan C, Byrne S, et al (2008) STOPP (Screening Tool of Older Person's Prescriptions) and START (Screening Tool to Alert doctors to
Right Treatment). Consensus validation. International Journal of Clinical Pharmacology, Therapy, and Toxicology, 46: 72-83.

Gareri P, De Fazio P, Russo E, et al (2008) The safety of clozapine in the elderly. Expert Opinion on Drug Safety, 7: 525-38.

Guenette MD, Powell V, Johnston K, et al (2013) Risk of neutropenia in a clozapine-treated elderly population. Schizophrenia Research, 148(1-3): 183-5.

Hack N, Fayad SM, Monari EH, et al (2014) An eight-year clinic experience with clozapine use in a Parkinson's disease clinic setting. PLoS ONE, 9(3): e91545.

Herbert Y, Meltzer MD, Alphs L (2003) Clozapine treatment for suicidality in schizophrenia: International Suicide Prevention Trial (InterSePT). Archives of General Psychiatry, 60: 82-91.

Joint Formulary Committee (2017) British National Formulary (74th edn). BMJ Group and Pharmaceutical Press.

Kane J, Honigfeld G, Singer J (1988) Clozapine for the treatment resistant schizophrenic: a double-blind comparison with chlorpromazine. Archives of General Psychiatry, 45: 789-96.

Klein C, Gordon J, Pollack L, et al (2003) Clozapine in Parkinson's disease psychosis: 5 year follow-up review. Clinical Neuropharmacology, 26: 8-11.

Kohen I, Lester P, Lam S (2010) Antipsychotic treatments for the elderly: efficacy and safety of aripiprazole. Neuropsychiatric Disease and Treatment, 6: 47-58.

Kuzuhara S (2001) Drug-induced psychotic symptoms in Parkinson's disease. Problems, management and dilemma. Journal of Neurology, 248(suppl 3): III28-31.

Lally J, Brook J, Dixon T, et al (2014) Ivabradine, a novel treatment for clozapine-induced sinus tachycardia: a case series. Therapeutic Advances Psychopharmacology, 4: 117-22.

Lally J, Docherty MJ, MacCabe JH (2016) Pharmacological interventions for clozapine-induced sinus tachycardia. Cochrane Database of Systematic Reviews, 6: CD011566 (doi: 10.1002/14651858.CD011566.pub2).

Lamberti JS, Olson D, Crilly JF, et al (2006) Prevalence of the metabolic syndrome among patients receiving clozapine. American Journal of Psychiatry, 163: 1273-6.

Lieberman JA, Stroup TS, McEvoy JP, et al (2005) Effectiveness of antipsychotic drugs in patients with chronic schizophrenia. New England Journal of Medicine, 353: 1209-23.

Medicines and Healthcare products Regulatory Agency (2017) Clozapine: Reminder of Potentially Fatal Risk of Intestinal Obstruction, Faecal Impaction, and Paralytic Ileus (https://www.gov.uk/drug-safety-update/ clozapine-reminder-of-potentially-fatal-risk-of-intestinal-obstruction-faecalimpaction-and-paralytic-ileus). MHRA.

Naranjo CA, Hermann N, Mittmann N (1995) Recent advances in geriatric psychopharmacology. Drugs and Aging, 7: 184-202.

National Institute for Health and Care Excellence (2014) Psychosis and Schizophrenia in Adults: Prevention and Management (Clinical Guideline CG178). NICE.

National Institute for Health and Care Excellence (2017) Parkinson's Disease in Adults (NICE Guideline NG71). NICE.

Paranthaman R, Baldwin R (2006) Survey of clozapine use by consultant old age psychiatrists. Psychatric Bulletin, 30: 410-2.

Parkinson Study Group (1999) Low-dose of clozapine for the treatment of drug-induced psychosis in Parkinson's disease. New England Journal of Medicine, 340: 757-63.

Parks J, Svendsen D, Singer P, et al (eds) (2006) Morbidity and Mortality in People with Serious Mental IIIness. National Association of State Mental Health Program Directors (http://www.nasmhpd.org/sites/ default/files/Mortality\%20and\%20Morbidity\%20Final\%20Report\%208. 18.08.pdf).

Pollack P, Tison F, Rascol 0 (2004) Clozapine in drug induced psychosis in Parkinson's disease: a randomised, placebo controlled study with open follow up. Journal of Neurology, Neurosurgery \& Psychiatry, 75: 689-95.

Praharaj SK, Arora M, Gandorta S (2006) Clozapine-induced sialorrhea: pathophysiology and management strategies. Psychopharmacology, 185: 265-73 
Pridan S, Swartz M, Baruch Y, et al (2015) Effectiveness and safety of clozapine in elderly patient with chronic resistant schizophrenia. International Psychogeriatrics, 27: 131-4.

Ronaldson KJ, Fitzgerald PB, Taylor AJ, et al (2011) A new monitoring protocol for clozapine induced myocarditis bases on an analysis of 75 cases and 94 controls. Australian and New Zealand Journal of Psychiatry, 45: 458-65.

Royal College of Psychiatrists (2015) Schizophrenia Key Facts: UK population. Royal College of Psychiatrists (http://www.rcpsych.ac.uk/healthadvice/problemsanddisorders/schizophreniakeyfacts.aspx).

Sajatovic M, Ramirez LF, Garver D, et al (1998) Clozapine therapy for older veterans. Psychiatric Services, 49: 340-4.

Salzman C (2005) Clinical Geriatric Psychopharmacology (4th edn). Lippincott Williams \& Wilkins.
Sanyal S, Van Tol HH (1997) Review the role of dopamine D4 receptors in schizophrenia and antipsychotic action. Journal of Psychiatric Research, 31: 219-32.

Scottish Government Model of Care Polypharmacy Working Group (2015) Polypharmacy Guidance: March 2015 (2nd edn). Scottish Government. (http://sign.ac.uk/assets/polypharmacy_guidance.pdf).

Taylor D, Patron C, Kapur S (2015) The Maudsley Prescribing Guidelines in Psychiatry (12th edn). Wiley-Blackwell.

Tiihonen J, Lönnqvist J, Wahlbeck K, et al (2009) 11-year follow-up of mortality in patients with schizophrenia: a population-based cohort study (FIN11 study). Lancet, 374: 620-27.

Tune LE, Salzman C (2003) Schizophrenia in late life. Psychiatric Clinics of North America, 26: 103-13.

\section{MCOs}

1 As regards psychosis in Parkinson's disease, it is not true that:

a clozapine is the drug of choice to treat the psychosis

b $20-30 \%$ of people with Parkinson's disease will develop psychotic symptoms

c thought disorder is the prominent symptom

d psychosis can be an iatrogenic side-effect of antiparkinsonian drugs

e guidelines advise reducing the dose of antiparkinsonian drugs to help with psychotic symptoms.

2 In clozapine prescribing for older patients:

a the starting dose can be increased in increments of $50 \mathrm{mg} / \mathrm{day}$

b there is a separate age-specific titration regime to follow c on the first day, the patient should be given a loading dose of $50 \mathrm{mg}$

4 The receptor implicated in orthostatic hypotension in clozapine use is:

$\mathrm{d}$ the mean therapeutic dose is $25-100 \mathrm{mg} /$ day

e the patient should have a full blood count (FBC) twice a week until the therapeutic dose is reached.

a $\mathrm{H}_{1}$

b alpha-1

c alpha-2

d $5-\mathrm{HT}_{10}$

e muscarinic

3 As regards pharmacokinetics and pharmacodynamics in older adults:

a there is an increase in volume distribution of drugs

b there is an increase in hepatic clearance

c there is an increase in renal clearance

$\mathrm{d}$ there is a decrease in gut motility

e there is increased absorption from the small intestine.
5 As regards clozapine-induced agranulocytosis:

a the incidence has consistently been found to be $3 \%$

$\mathrm{b}$ the risk increases with advancing age

c the risk is higher in men

d it is associated with higher prescribed doses

$\mathrm{e}$ it is associated with higher plasma levels. 